\title{
Crack arrest model for a piezoelectric plate - A generalised Dugdale model
}

\author{
R R BHARGAVA ${ }^{1}$ and NAMITA SAXENA \\ Department of Mathematics, Indian Institute of Technology, Roorkee 247667 , \\ India \\ e-mail: \{rajrbfma,mitandma\}@iitr.ernet.in
}

MS received 9 July 2005; revised 6 June 2006

\begin{abstract}
The constant search for new materials has provided impetus to research in piezoelectric materials. An anti-plane problem for a cracked unbounded twodimensional poled piezoelectric plate has been investigated. The crack rims open on account of shear mechanical forces applied at the remote boundary and inplane electric displacement field prescribed at the infinite boundary. Thus the crack yields both mechanically and electrically. Consequently, a plastic zone and a saturation zone protrude ahead of each tip of the crack. These developed zones are in turn closed by applying yield point shear stress at the rims of plastic zone and normal closing saturation limit displacement on the rims of saturation zones. Two cases are investigated when (i) the developed saturation zone length exceeds that of the developed plastic zone, and (ii) saturation zone length is smaller than that of the plastic zone. Fourier integral transform method is used in each case to obtain the length of plastic zone and saturation zone. Closed form analytic expressions are obtained in each case. Crack opening displacement and potential drop across the rims of the crack are also obtained. The effect of mechanical loads on crack closure in the presence of electric field is investigated and vice-versa. Also energy release rate expressions are obtained for both the cases.
\end{abstract}

Keywords. Piezoelectric plate; energy release rate; plastic zone; saturation zone; crack opening displacement; crack opening potential.

\section{Introduction}

Piezoelectric materials by now have proven their utility in technological and industrial applications. Piezoelectric material being the future material for sophisticated areas there is a natural interest of the researchers in studying the fracture behaviour and fracture mechanics of these materials. Gao \& Fan (1999) obtained exact solution of the plane problem for an infinite piezoelectric media with an elliptic cavity under the permeable boundary condition. Narita \& Shindo (2001) studied a Mode-I crack growth rate for a finite crack in a narrow transversely isotropic piezoelectric ceramic body under tensile loading. McMeeking (2004) developed a theoretical fracture mechanics for brittle piezoelectric and dielectric materials consistent with standard features of elasticity and dielectricity. More recently 
Wang \& Yu (2001) studied the Mode-I transient response of a finite crack weakening piezoelectric ceramic under infinite mechanical and electrical impacts. More recently Bhargava \& Saxena (2005) have obtained solution for a crack arrest problem for Mode-I case.

\section{Fundamental formulation}

A poled unbounded piezoelectric plate occupying xoy-plane is cut along a crack, of length $2 a$, which lies along the $x$-axis in cartesian coordinate system $(x, y)$ with its rims along the $x$-axis. The crack is perpendicular to the poling axis, i.e. the $y$-axis. For plane strain case

$$
u_{x}=0, u_{y}=w(x, y),
$$

and in-plane electrical field components are

$$
E_{x}=0, E_{y}=E_{y}(x, y),
$$

where $u_{i}, E_{i}(i=x, y)$ are elastic displacement and electric field components. The governing equations have following form.

$$
\begin{aligned}
\sigma_{x x} & =c_{13} \frac{\partial w}{\partial y}+e_{31} \frac{\partial \phi}{\partial y}, \sigma_{x y}=c_{44} \frac{\partial w}{\partial x}+e_{15} \frac{\partial \phi}{\partial x}, \sigma_{y y}=c_{33} \frac{\partial w}{\partial y}+e_{33} \frac{\partial \phi}{\partial y}, \\
D_{x} & =e_{15} \frac{\partial w}{\partial x}-\varepsilon_{11} \frac{\partial \phi}{\partial x}, D_{y}=e_{33} \frac{\partial w}{\partial y}-\varepsilon_{33} \frac{\partial \phi}{\partial y},
\end{aligned}
$$

where $E_{i}=-(\partial \phi / \partial i)(i=x, y)$ and $\phi$ denotes the electric potential $c_{i j}, e_{i j}$ and $\varepsilon_{i j}$ are the elastic, piezoelectric and dielectric constants of the plate and $\sigma_{i j}$ and $D_{i}(i, j=x, y)$ denote stress components and displacement components respectively.

Constitutive equations are written as

$$
\begin{aligned}
& c_{44} \frac{\partial^{2} w}{\partial x^{2}}+c_{33} \frac{\partial^{2} w}{\partial y^{2}}+e_{15} \frac{\partial^{2} \phi}{\partial x^{2}}+e_{33} \frac{\partial^{2} \phi}{\partial y^{2}}=0, \\
& e_{15} \frac{\partial^{2} w}{\partial x^{2}}+e_{33} \frac{\partial^{2} w}{\partial y^{2}}-\varepsilon_{11} \frac{\partial^{2} \phi}{\partial x^{2}}-\varepsilon_{33} \frac{\partial^{2} \phi}{\partial y^{2}}=0 .
\end{aligned}
$$

On a qualitative basis, only three independent material constants $M, e$ and $\varepsilon$ may be used to represent elastic, piezoelectric and dielectric properties of the material. Consequently, the set of equations (1) and (2) simplify to the following electroelastic model.

Equations (2) yields

$$
\nabla^{2} w=0, \text { and } \nabla^{2} \phi=0,
$$

and (1) reduce to

$$
\begin{aligned}
& \sigma_{x x}=M \frac{\partial w}{\partial y}+e \frac{\partial \phi}{\partial y}, \quad \sigma_{x y}=M \frac{\partial w}{\partial x}+e \frac{\partial \phi}{\partial x}, \quad \sigma_{y y}=M \frac{\partial w}{\partial y}+e \frac{\partial \phi}{\partial y} \\
& D_{x}=e \frac{\partial w}{\partial x}-\varepsilon \frac{\partial \phi}{\partial x}, \quad D_{y}=e \frac{\partial w}{\partial y}-\varepsilon \frac{\partial \phi}{\partial y} .
\end{aligned}
$$


Here, $\nabla^{2}=\frac{\partial^{2}}{\partial x^{2}}+\frac{\partial^{2}}{\partial y^{2}}$ is the two-dimensional Laplacian operator and $w$ and $\phi$ are the in-plane displacement and electric potential respectively.

Equations (3) are solved for finding the solution of a particular problem under given boundary conditions. Stresses and electric displacements are then calculated by (4) and (5). Fundamental solutions of (3) are obtained by employing the Fourier transform to the equations. Writing the equations in vector form as

$$
\nabla^{2} \tilde{u}=0
$$

where $\tilde{u}(z)=\left\{\begin{array}{l}w \\ \phi\end{array}\right\}$, and taking the Fourier transform of (6), denoting it by $U(s, y)$, the following is obtained,

$$
\frac{\partial^{2} U}{\partial y^{2}}-s^{2} U=0
$$

Similarly, denoting by $\tilde{t}(x, y)=\left\{\begin{array}{c}\sigma_{x y} \\ D_{y}\end{array}\right\}$ and taking Fourier transforms of the second equations of each set (4) and (5), we obtain

$$
T(s, y)=\left(\begin{array}{cc}
M & e \\
e & -\varepsilon
\end{array}\right) \frac{\partial U(s, y)}{\partial y},
$$

where $T(s, y)$ denotes the Fourier transform of $t(x, y)$ with respect to $x$.

The solution of (7) may be written as

$$
U(s, y)= \begin{cases}e^{s y} \tilde{C}, & s<0, \\ e^{-s y} \tilde{F}, & s>0 .\end{cases}
$$

Consequently with the help of (9), (8) may be written as

$$
T(s, y)= \begin{cases}\left(\begin{array}{cc}
M & e \\
e & -\varepsilon
\end{array}\right) s U(s, y), & s<0, \\
-\left(\begin{array}{cc}
M & e \\
e & -\varepsilon
\end{array}\right) s U(s, y), & s>0,\end{cases}
$$

where $\tilde{C}$ and $\tilde{F}$ are two component vectors.

In (8) substituting $y=0$, we get

$$
T(s, 0)=s\left(\begin{array}{cc}
M & e \\
e & -\varepsilon
\end{array}\right) U(s, 0) .
$$

From the condition of continuity of traction at $y=0$, we obtain

$$
U^{+}(s, 0)+U^{-}(s, 0)=0 .
$$

If the jump in displacement and electric potential, at $y=0$, be defined as

$$
\Delta \tilde{u}(x)=\tilde{u}^{+}(x, 0)-\tilde{u}^{-}(x, 0),
$$


and the dislocation and dipole density vector is given by

$$
\tilde{f}(x)=\left\{\begin{array}{l}
f_{1}(x) \\
f_{2}(x)
\end{array}\right\}=\frac{\mathrm{d}}{\mathrm{d} x} \tilde{u}(x),
$$

then taking Fourier transform of (12) and (13) leads to

$$
\Delta U(s)=\tilde{U}^{+}(s)-\tilde{U}^{-}(s)=\frac{i}{s} \int_{-a}^{a} \tilde{f}(x) e^{i s x} \mathrm{~d} x,
$$

and taking Fourier inverse transform of (10) and combining (11) and (14) the following are obtained,

$$
\tilde{t}(x)=\frac{1}{\pi}\left(\begin{array}{cc}
M & e \\
e & -\varepsilon
\end{array}\right) \int_{-a}^{a} \frac{\tilde{f}(t)}{t-x} \mathrm{~d} t=\left(\begin{array}{c}
t_{1}^{0}(x) \\
t_{2}^{0}(x)
\end{array}\right),
$$

where $t_{1}^{0}(x)$ and $t_{2}^{0}(x)$ are prescribed mechanical and electrical boundary conditions.

\section{The problem}

An unbounded infinite piezoelectric plate, occupying the xoy-plane, is taken to be poled along the $o y$-axis. The plate is cut along an internal hairline straight crack, $L$, lying along $o x$-axis and occupies the interval $[-a, a]$. This configuration is subjected to uniform and constant (i) mechanical shear stress, $\tau_{\infty}$ and (ii) in-plane electrical displacement, $D_{\infty}$, at infinite boundary of the plate. Consequently rims of the crack open in anti-plane deformations, yielding the crack both electrically and mechanically. Under small-scale yield, it is assumed that electrical polarization is situated along a line segment in front of the each tip of the crack. Similar to the assumption in the Dugdale model, a plastic zone is assumed to protrude at each tip of the crack, along a line segment in front of the crack. The developed plastic zones, $r_{1}$ and $r_{2}$, occupy in the interval $[-b,-a]$ and $[a, b]$ along the $o x$-axis respectively.

The developed saturation zones, $s_{1}$ and $s_{2}$, occupy the interval $[-l,-a]$ and $[a, l]$ along the $o x$-axis respectively. These developed saturation and plastic zones, in turn, are arrested by applying a normal, cohesive saturation limit electrical displacement and yield point shear stress plate, at the rims of developed saturation and plastic zones, respectively. Thus the crack is stopped from opening further. The configuration is schematically depicted in figure 1. Two cases are investigated (i) when length of saturation zone is bigger than that of plastic zone and (ii) when plastic zone length is bigger than that of saturation zone.

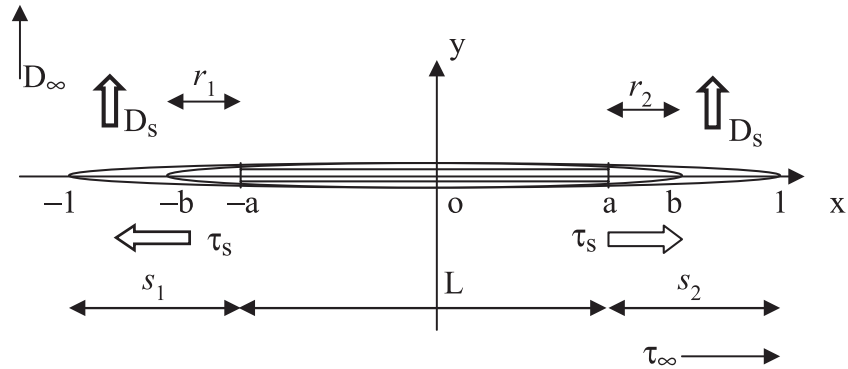

Figure 1. Schematic representation of configuration of problem. 


\subsection{Case I: Mathematical model when size of saturation zone is bigger than plastic zone}

An unbounded poled piezoelectric plate occupies the entire xoy-plane. The plate is cut along a crack of length $2 l$ when occupies the interval $[-l, l]$ on the $o x$-axis. The rims of the crack are perpendicular to the poling direction. The boundary conditions of the problem are as below,

(a) for $|x|<a, \sigma_{x y}^{0}=\tau_{\infty}=t_{1}^{0}$ and for $a<|x|<b, \sigma_{x y}^{0}=-\tau_{\infty}+\tau_{s}=t_{1}^{0}$;

(b) for $|x|<a, D_{y}^{0}=-D_{\infty}=t_{2}^{0}$ and for $a<|x|<l, D_{y}^{0}=-D_{\infty}+D_{s}=t_{2}^{0}$;

(c) displacement and electric potential are single-valued around crack;

(d) conditions of continuity of dislocation and dipole density along $x$-axis requires

$$
f_{1}(x)=0 \text { for }|x|>b, f_{2}(x)=0 \text { for }|x|>l .
$$

3.1a Solution of case I: The solution of the problem is obtained by solving (15) together with the given boundary conditions. The following equations are obtained when boundary condition (d) as above is applied to (15) which yields

$$
\begin{aligned}
& \quad \frac{1}{\pi} \int_{-b}^{b} \frac{f_{1}(t)}{t-x} \mathrm{~d} t=\frac{1}{M \varepsilon+e^{2}}\left(-\varepsilon t_{1}^{0}+e t_{2}^{0}\right), \\
& \text { and } \frac{1}{\pi}\left(l^{2}-x^{2}\right)^{1 / 2} \int_{-l}^{l} \frac{t_{2}^{0}}{(t-x)\left(l^{2}-t^{2}\right)^{1 / 2}} \mathrm{~d} t=e f_{1}(x)-\varepsilon f_{2}(x) .
\end{aligned}
$$

Since the function $f_{1}(x)$ is non-singular at $|x|=0,(16)$ has a solution provided

$$
\frac{1}{M \varepsilon+e^{2}} \int_{-b}^{b} \frac{-\varepsilon t_{1}^{0}+e t_{2}^{0}}{\left(b^{2}-t^{2}\right)^{1 / 2}}, \mathrm{~d} t=0
$$

which on evaluation by substituting values of $t_{1}^{0}$ and $t_{2}^{0}$ yields the equation for determining plastic zone length

$$
b=a \sec \left\{\frac{\pi}{2}\left(\frac{e D_{\infty}-\varepsilon \tau_{\infty}}{e D_{s}-\varepsilon \tau_{s}}\right)\right\} .
$$

Using, (19) the solution of (18) may be written as

$$
\begin{aligned}
f_{1}(x)= & \frac{-\varepsilon \tau_{s}+e D_{s}}{\pi\left(M \varepsilon+e^{2}\right)}\left[\cosh ^{-1}\left\{\frac{b^{2}-x^{2}}{(a-x) b}-\frac{x}{b}\right\}-\cosh ^{-1}\left\{\frac{x^{2}-b^{2}}{(a+x) b}-\frac{x}{b}\right\}\right], \\
& \text { for }|x|<b .
\end{aligned}
$$

Similarly, (17) could be solved if

$$
\int_{-l}^{l} \frac{t_{2}^{0}}{\left(l^{2}-t^{2}\right)^{1 / 2}} \mathrm{~d} t=0 .
$$

Substituting the value of $t_{2}^{0}$ from the boundary condition (b) and computing, the following transdental equation is obtained to determine saturation zone length

$$
l=a \sec \left(\frac{\pi}{2} \frac{D_{\infty}}{D_{s}}\right) .
$$


Equation (18) in conjugation with (22) yields

$$
f_{2}(x)=-\frac{D_{s}}{\varepsilon \pi}\left[\cosh ^{-1}\left\{\frac{l^{2}-x^{2}}{(a-x) l}-\frac{x}{l}\right\}-\cosh ^{-1}\left\{\frac{x^{2}-l^{2}}{(a+x) l}-\frac{x}{l}\right\}\right]+\frac{e}{\varepsilon} f_{1}(x)
$$

Thus the required functions $f_{2}(x)$ and $f_{1}(x)$ are completely determined for case $\mathrm{I}$.

\subsection{Case II: Mathematical model when size of plastic zone is bigger than the saturation zone}

A poled piezoelectric infinite plate occupies the xoy-plane. A finite hairline, straight crack occupying the interval $[-a, a]$ along the $o x$-axis, weakens the plate.

The boundary conditions of the problem are the following

(i) $-\tau_{\infty}=t_{1}^{0}$ if $|x|<a$ and $-\tau_{\infty}+\tau_{s}=t_{1}^{0}$ if $a<|x|<b<l$;

(ii) $-D_{\infty}=t_{2}^{0}$, if $|x|<a$ and $-D_{\infty}+D_{s}=t_{2}^{0}$ if $a<|x|<b$;

(iii) Single-valuedness condition for displacement and electric potential is satisfied around the crack;

(iv) $f_{2}(x)=0$, for $|x|>b$.

3.2a Solution of case II: Potential $f_{2}(x)$ is determined using (15) and boundary conditions (i to iv) by the integral equation,

$$
\int_{-b}^{b} \frac{f_{2}(x)}{t-x} \mathrm{~d} x=\frac{\pi}{M \varepsilon+e^{2}}\left\{e t_{1}^{0}+M t_{2}^{0}\right\}, \text { for }|x|<b .
$$

Equation (24) has a solution provided

$$
\int_{-b}^{b} \frac{e t_{1}^{0}+M t_{2}^{0}}{\left(b^{2}-t^{2}\right)^{1 / 2}} \mathrm{~d} t=0
$$

which yields the nonlinear equation below to determine the size of saturation zone

$$
b=a \sec \left\{\frac{\pi}{2}\left(\frac{M D_{\infty}+e \tau_{\infty}}{M D_{s}+e \tau_{s}}\right)\right\} .
$$

Equation (24) together with (26) determines $f_{2}(x)$ from the relation

$$
\begin{aligned}
f_{2}(x)= & \frac{e \tau_{s}+M D_{s}}{\pi\left(M \varepsilon+e^{2}\right)}\left[\cosh ^{-1}\left\{\frac{b^{2}-x^{2}}{(a-x) b}-\frac{x}{b}\right\}-\cosh ^{-1}\left\{\frac{x^{2}-b^{2}}{(a+x) b}-\frac{x}{b}\right\}\right], \\
& \text { for }|x|<b .
\end{aligned}
$$

Similarly, the solution of (15) together with boundary condition (i) determines the function $f_{1}(x)$ through integral equation

$$
M f_{1}(x)=-e f_{2}(x)-\frac{1}{\pi}\left(l^{2}-x^{2}\right)^{1 / 2} \int_{-l}^{l} \frac{t_{1}^{0}}{(t-x)\left(l^{2}-t^{2}\right)^{1 / 2}} \mathrm{~d} t .
$$


Equation (28) has a solution provided

$$
\int_{-l}^{l} \frac{t_{1}^{0}}{\left(l^{2}-t^{2}\right)^{1 / 2}} \mathrm{~d} t=0
$$

Evaluation of this integral yields,

$$
l=a \sec \left\{\frac{\pi}{2} \frac{\tau_{\infty}}{\tau_{s}}\right\}
$$

Equations (28), (29) and (27) give the value of function $f_{1}(x)$ as

$$
f_{1}(x)=\frac{\tau_{s}}{\pi M}\left[\cosh ^{-1}\left\{\frac{l^{2}-x^{2}}{(a-x) l}-\frac{x}{l}\right\}-\cosh ^{-1}\left\{\frac{x^{2}-l^{2}}{(a+x) l}-\frac{x}{l}\right\}\right]-\frac{e}{M} f_{2}(x)
$$

Thus case II is completely solved.

\section{Crack opening displacement and crack opening potential}

\subsection{Case I}

The crack opening displacement (COD) $\Delta w^{I}(x)$ and crack opening potential drop $\Delta \phi^{I}(x)$ across the faces of the crack are given by

$$
\begin{aligned}
\Delta w^{I}(x)= & -\int_{-b}^{x} f_{1}(x) \mathrm{d} x \\
= & \frac{e D_{s}-\varepsilon \tau_{s}}{\pi\left(M \varepsilon+e^{2}\right)}\left[(a-x) \cosh ^{-1}\left\{\frac{b^{2}-x^{2}}{(a-x) b}-\frac{x}{b}\right\}\right. \\
& \left.+(a+x) \cosh ^{-1}\left\{\frac{x^{2}-b^{2}}{(a+x) b}-\frac{x}{b}\right\}\right], \text { for }|x|<b \\
\Delta \phi^{I}(x)= & -\frac{D_{s}}{\pi \varepsilon}\left[(a-x) \cosh ^{-1}\left\{\frac{l^{2}-x^{2}}{(a-x) l}-\frac{x}{l}\right\}\right. \\
& \left.+(a+x) \cosh ^{-1}\left\{\frac{x^{2}-l^{2}}{(a+x) l}-\frac{x}{l}\right\}\right]+\frac{e}{\varepsilon} \Delta w^{I}(x) .
\end{aligned}
$$

Consequently, the COD and crack opening potential (COP) at the tip $x=a$ of actual tip of the crack are given by

$$
\begin{aligned}
& \Delta w^{I}(a)=\frac{2 a}{\pi\left(M \varepsilon+e^{2}\right)}\left(-\varepsilon \tau_{s}+e D_{s}\right) \ln \left[\sec \frac{\pi}{2}\left(\frac{e D_{\infty}-\varepsilon \tau_{\infty}}{e D_{s}-\varepsilon \tau_{s}}\right)\right], \\
& \Delta \phi^{I}(a)=-\frac{2 a}{\pi \varepsilon} D_{s} \ln \left[\sec \left\{\frac{\pi}{2} \frac{D_{\infty}}{D_{s}}\right\}\right]+\frac{e}{\varepsilon} \Delta w^{I}(a),
\end{aligned}
$$

where the superscript I denotes the quantities pertaining to case I. 


\subsection{Case II}

The potential drop across crack rims for this case is given by

$$
\begin{aligned}
\Delta \phi^{I I}(x)= & \frac{M D_{s}+e \tau_{s}}{\pi\left(M \varepsilon+e^{2}\right)}\left[(a-x) \cosh ^{-1}\left\{\frac{b^{2}-x^{2}}{(a-x) b}-\frac{x}{b}\right\}\right. \\
& \left.+(a+x) \cosh ^{-1}\left\{\frac{x^{2}-b^{2}}{(a+x) b}-\frac{x}{b}\right\}\right], \text { for }|x|<b
\end{aligned}
$$

The superscript II denotes the parameters pertaining to case II. The crack rim opening displacement is

$$
\begin{aligned}
\Delta w^{I I}(x)= & \frac{\tau_{s}}{\pi M}\left[(a-x) \cosh ^{-1}\left\{\frac{l^{2}-x^{2}}{(a-x) l}-\frac{x}{l}\right\}-\frac{e \pi}{\tau_{s}} \Delta \phi^{I I}(x)\right. \\
& \left.+(a+x) \cosh ^{-1}\left\{\frac{x^{2}-l^{2}}{(a+x) l}-\frac{x}{l}\right\}\right] .
\end{aligned}
$$

Finally, COP and COD at the crack tip $x=a$ are given by

$$
\begin{aligned}
\Delta \phi^{I I}(a) & =\frac{2 a}{\pi} \frac{e \tau_{s}+M D_{s}}{\left(M \varepsilon+e^{2}\right)} \ln \left[\sec \frac{\pi}{2}\left(\frac{M D_{\infty}+e \tau_{\infty}}{M D_{s}+e \tau_{s}}\right)\right], \\
\text { and } \Delta w^{I I}(a) & =\frac{2 a}{\pi} \frac{\tau_{s}}{M} \ln \left[\sec \left\{\frac{\pi}{2} \frac{\tau_{\infty}}{\tau_{s}}\right\}\right]-\frac{e}{M} \Delta \phi^{I I}(a)
\end{aligned}
$$

respectively.

\section{Energy release rate}

\subsection{Case I}

Energy release rate, $J_{a}^{I}$, at the crack tip $x=a$ for case $\mathrm{I}$ is given by

$$
J_{a}^{I}=\tau_{s} \Delta w^{I}(a)+D_{s} \Delta \phi^{I}(a) .
$$

Substituting values of $\Delta w^{I}(a)$ and $\Delta \phi^{I}(a)$ from (33 and 34) and after some simplification, we get

$$
\begin{aligned}
J_{a}^{I}= & \frac{2 a}{\pi \varepsilon}\left[\frac{1}{\left(M \varepsilon+e^{2}\right)}\left(e^{2} D_{s}^{2}-\varepsilon^{2} \tau_{s}^{2}\right) \cosh ^{-1}\left\{\frac{a^{2}+b^{2}}{2 a b}\right\}\right. \\
& \left.-D_{s}^{2} \cosh ^{-1}\left\{\frac{a^{2}+l^{2}}{2 a l}\right\}\right] .
\end{aligned}
$$

\subsection{Case II}

Similarly energy release rate, $J_{a}^{I I}$, for the case II, at the crack tip $x=a$ is given by,

$$
J_{a}^{I I}=\tau_{s} \Delta w^{I I}(a)+D_{s} \Delta \phi^{I I}(a),
$$


Table 1. Material constants for piezoelectric ceramics.

\begin{tabular}{lcc}
\hline Ceramic & PZT-4 & $\mathrm{BaTiO}_{3}$ \\
\hline$M\left(10^{10} \mathrm{~N} / \mathrm{m}^{2}\right)$ & $2 \cdot 56$ & 4.4 \\
$\varepsilon\left(10^{-10} \mathrm{~N} / \mathrm{m}^{2}\right)$ & $64 \cdot 634$ & $98 \cdot 722$ \\
$e\left(\mathrm{C} / \mathrm{m}^{2}\right)$ & $12 \cdot 7$ & 11.4 \\
\hline
\end{tabular}

values of $\Delta w^{I I}(a)$ and $\Delta \phi^{I I}(a)$ are substituted in the above relation from (37) and (38), and rearranging the terms we have

$$
\begin{aligned}
J_{a}^{I I}= & \frac{2 a}{\pi M}\left[\frac{1}{\left(M \varepsilon+e^{2}\right)}\left(M^{2} D_{s}^{2}-e^{2} \tau_{s}^{2}\right) \cosh ^{-1}\left\{\frac{a^{2}+b^{2}}{2 a b}\right\}\right. \\
& \left.+\tau_{s}^{2} \cosh ^{-1}\left\{\frac{a^{2}+l^{2}}{2 a l}\right\}\right] .
\end{aligned}
$$

\section{Case study}

Behaviour of electrical displacement, crack opening displacement, crack opening potential drop and energy release rate with respect to plastic zone length and saturation zone length have been investigated for PZT-4 and $\mathrm{BaTiO}_{3}$ piezoelectric ceramics. The values of material constants taken are shown in table 1 .

\subsection{Case I}

Figure 2 depicts the variation of the ratio of the electrical displacements prescribed at infinity to the saturation limit displacement, $D_{\infty} / D_{s}$, with respect to plastic zone length to half crack length ratio. It is observed that as plastic zone length increases the electric displacement ratio also increases, while it decreases for negative increase in value of ratio $D_{\infty} / \tau_{\infty}$. The variations has been plotted for two materials PZT-4 and $\mathrm{BaTiO}_{3}$ for the value of the ratio $D_{\infty} / \tau_{\infty}$ varying between $-4 \times 10^{-10}$ and $4 \times 10^{-10}$.

Variation of electrical displacements ratio with respect to saturation zone length is plotted in figure 3. It is noted as the saturation zone length increases, the displacement ratio also increases. It may be noted that saturation zone is independent of material constants.

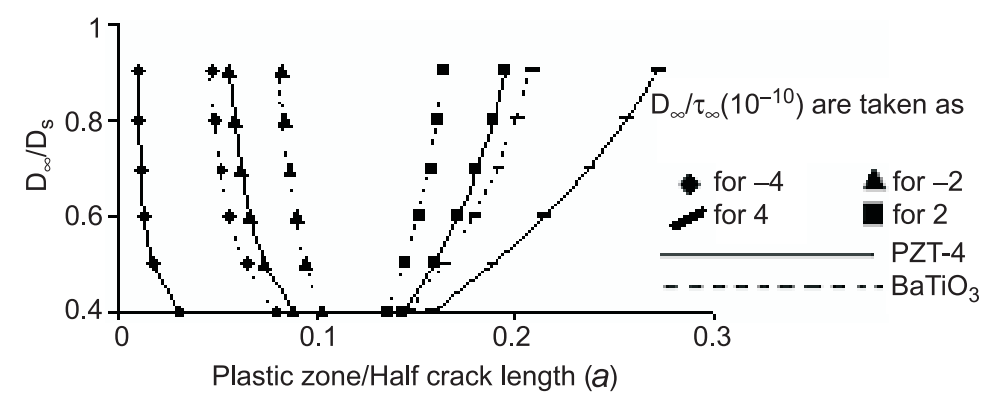

Figure 2. Variation of $D_{\infty} / D_{s}$ versus plastic zone to half crack length $(a)$ ratio. 


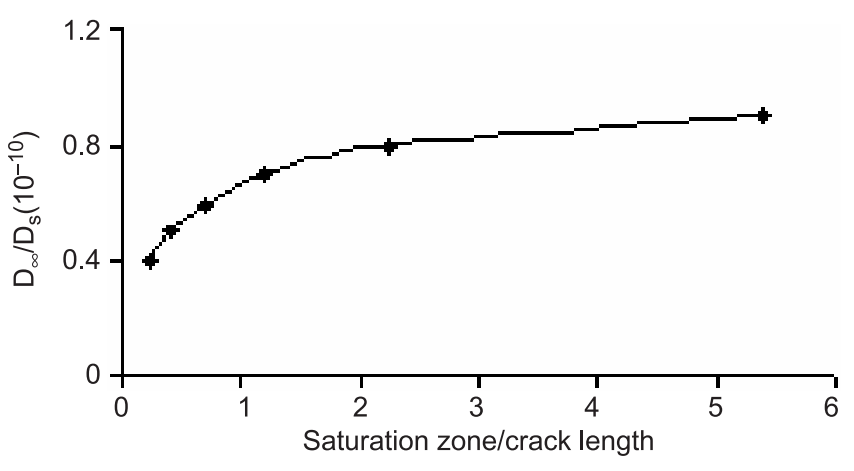

Figure 3. Variation of $D_{\infty} / D_{s}$ versus saturation zone to half crack length $(a)$ ratio.

Figures 4 and 5 show the variation of crack opening displacement, at the tip of the crack, with respect to plastic zone length to half crack length ratio. Figure 4 shows the variation for PZT-4 and figure 5 shows the same variation for $\mathrm{BaTiO}_{3}$. Crack rims open more for a bigger plastic zone length. COD at the crack tip is plotted for different crack lengths. Further it is noted that for greater crack length the crack opens more. It may further be noted that the crack rims open more for PZT- 4 as compared to that in case of $\mathrm{BaTiO}_{3}$ under similar conditions.

Behaviour of potential drop at the crack tip vis-a-vis plastic zone length is plotted in figures 6 and 7 for PZT- 4 and $\mathrm{BaTiO}_{3}$ respectively. For a fixed crack length the potential drop decreases as the plastic zone length is increased; near the 0.15 ratio value of plastic zone to crack length, the potential drop is zero for both the materials and it then becomes negative for further increase in plastic zone length. It is also observed as the crack length is increased, the potential drop at the crack tip also increases.

Energy release rate variation with respect to plastic zone length is shown as a graph in figures 8 and 9 for ceramics PZT-4 and $\mathrm{BaTiO}_{3}$ respectively. For a bigger crack length there is more energy release as compared to a smaller crack length for each of the ceramics.

\subsection{Case II}

Variation of $\tau_{\infty} / \tau_{s}$ vis-à-vis plastic zone length to half crack length is shown in figure 10 , for a fixed crack length. It is observed as plastic zone increases the requirement of load $\tau_{\infty} / \tau_{s}$ to arrest the plastic zone also increases.

Figure 11 depicts the behaviour of $\tau_{\infty} / \tau_{s}$ with respect to saturation zone to half crack length ratio for the variation of $D_{\infty} / \tau_{s}$ ratio varying between $-6 \times 10^{-10}$ to $6 \times 10^{-10}$. It is observed that for all values of $D_{\infty} / \tau_{s}$ ratio the $\tau_{\infty} / \tau_{s}$ remains positive and increases. It is further noted that for smaller saturation zone lengths, $D_{\infty} / \tau_{s}$ is positive, while for larger saturation zone lengths ratio the $D_{\infty} / \tau_{s}$ becomes negative.

Normalized crack opening displacement, $w$, variation is plotted in figures 12 and 13 versus saturation zone length for the ceramics PZT-4 and $\mathrm{BaTiO}_{3}$, and for various crack lengths. It is observed that as crack length increases the relative displacement of the crack faces is greater.

Behaviour of crack opening potential drop (COP) with respect to saturation zone is graphically shown in figures 14 and 15 for PZT- 4 and $\mathrm{BaTiO}_{3}$ respectively. Both ceramics show similar type of behaviour. It is also to be noted that for positive values of $\tau_{\infty} / \tau_{s} \mathrm{COP}$ increases while for negative values of $\tau_{\infty} / \tau_{s}$, it decreases for both the ceramics. 

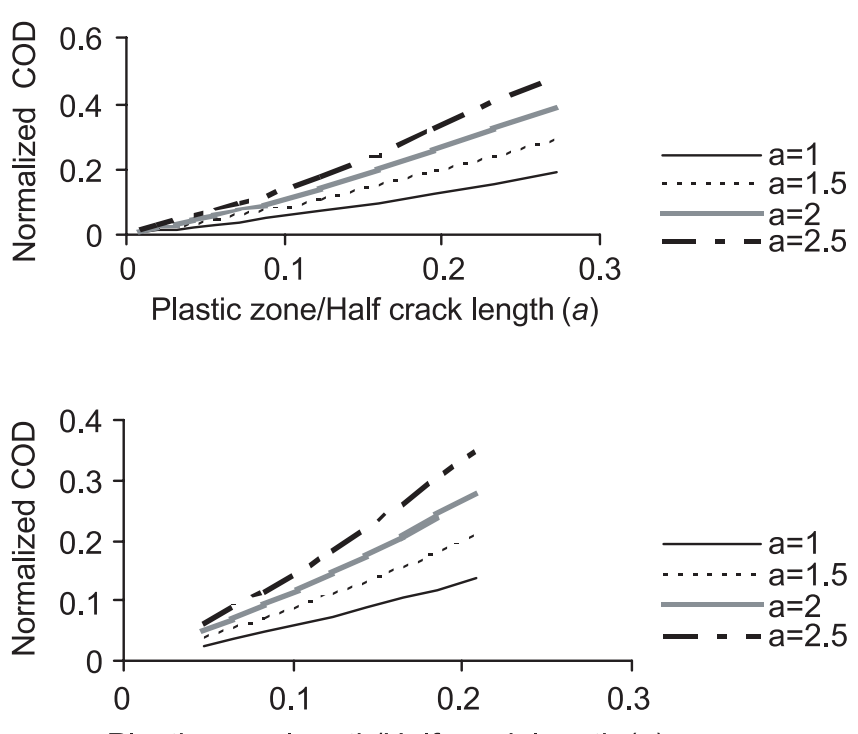

Plastic zone length/Half crack length (a)

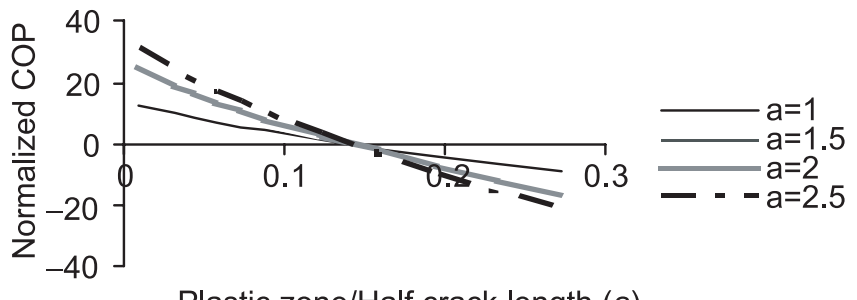

Plastic zone/Half crack length (a)

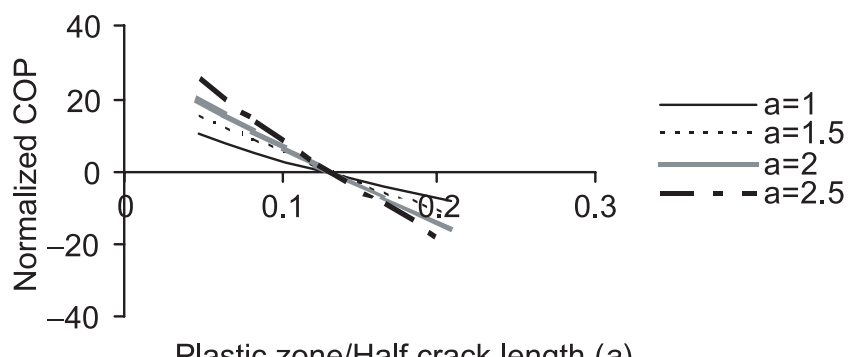

Plastic zone/Half crack length (a)

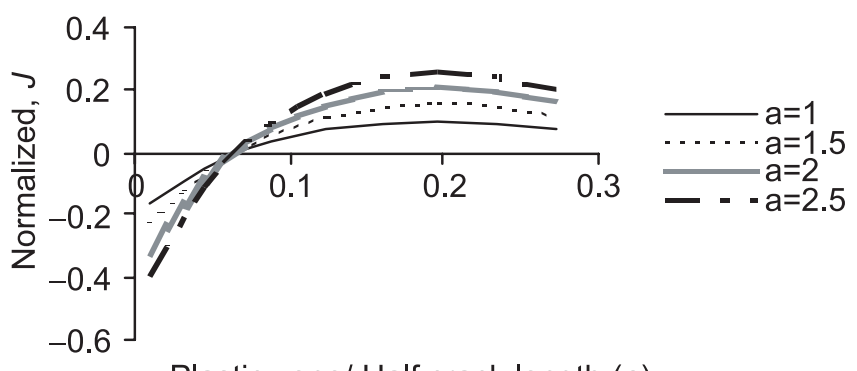

Plastic zone/ Half crack length (a)
Figure 4. Variation of normalized crack opening displacement (COD) for PZT-4.

Figure 5. Variation of normalized crack opening displacement (COD) for $\mathrm{BaTiO}_{3}$.

Figure 6. Variation of normalized potential drop (COP) for PZT-4.

Figure 7. Variation of normalized potential drop (COP) for $\mathrm{BaTiO}_{3}$.

Figure 8. Variation of normalized energy release rate, $J$, for PZT-4. 

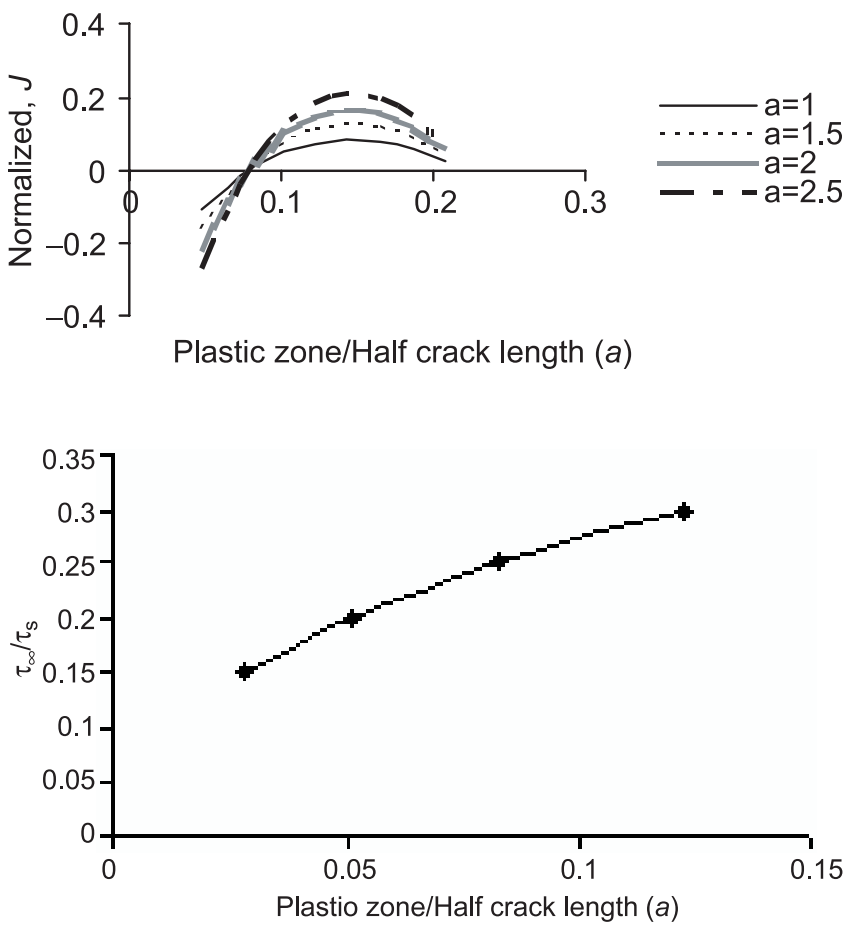

Figure 9. Variation of normalized energy release rate, $J$, for $\mathrm{BaTiO}_{3}$.

Figure 10. Variation of $\tau_{\infty} / \tau_{s}$ versus plastic zone to half crack length $(a)$ ratio.

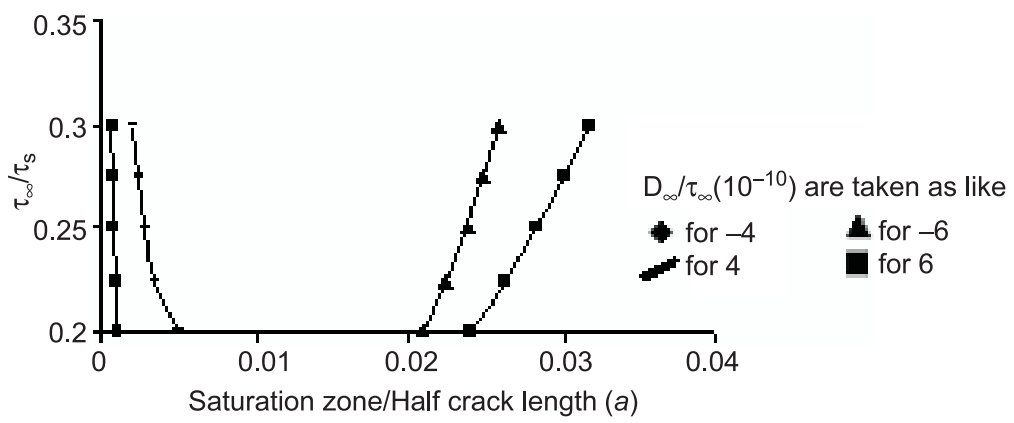

Figure 11. Variation of $\tau_{\infty} / \tau_{s}$ versus ratio of saturation zone to half crack length $(a)$ ratio.

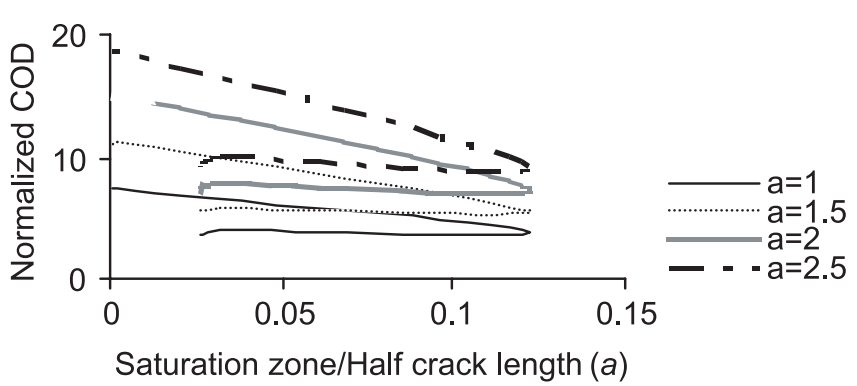

Figure 12. Variation of normalized crack opening displacement (COD) for PZT-4. 


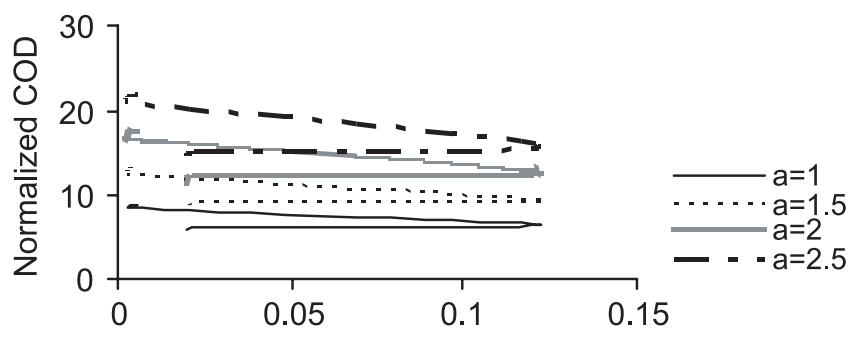

Saturation zone/Half crack length (a)

Figure 13. Variation of normalized crack opening displacement (COD) for $\mathrm{BaTiO}_{3}$.

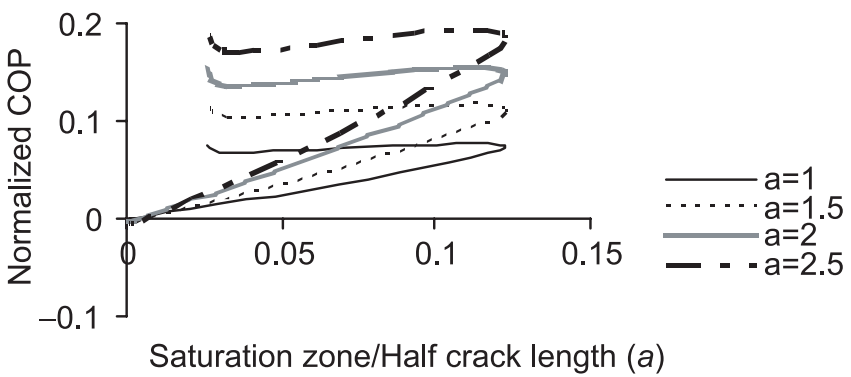

Figure 14. Variation of normalized potential drop (COP) for PZT-4.

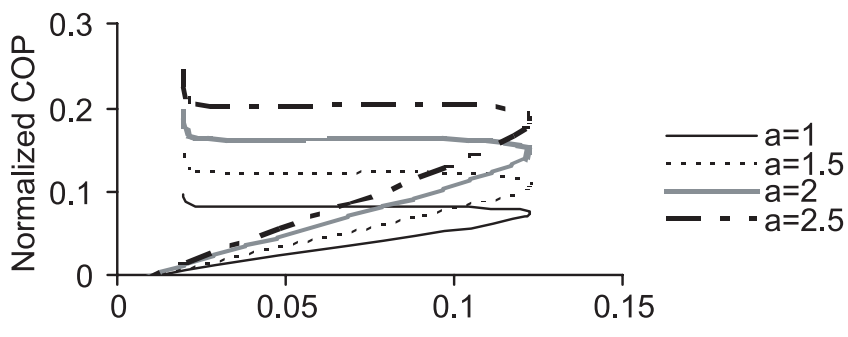

Figure 15. Variation of normalized potential drop (COP) for Saturation zone/Half crack length (a) $\mathrm{BaTiO}_{3}$.

Energy release rate behaviour is shown in figures 16 and 17 for PZT-4 and $\mathrm{BaTiO}_{3}$, with respect to saturation zone for $D_{\infty} / \tau_{s}$ ratios varying between $-6 \times 10^{-10}$ and $6 \times 10^{-10}$. The energy release reduces for all crack lengths and energy release rate becomes zero before becoming negative. It is to be noted that for bigger crack lengths, more energy is released.

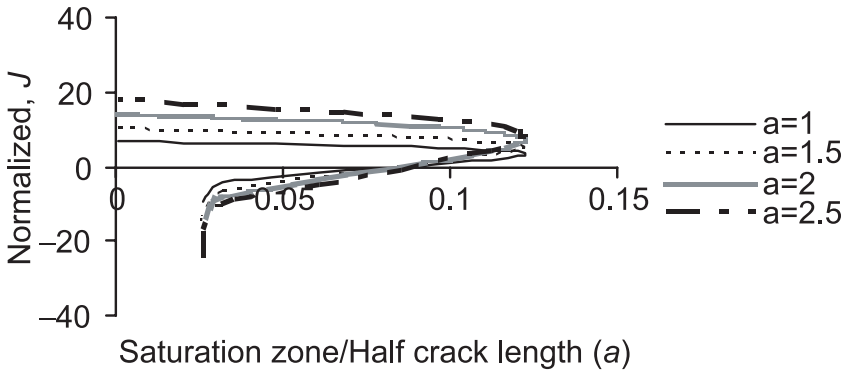

Figure 16. Variation of normalized energy release rate, $J$, for PZT-4. 


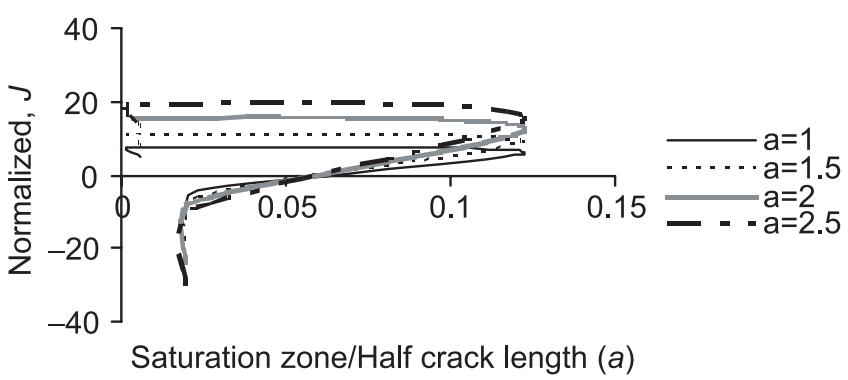

Figure 17. Variation of normalized energy release rate, $J$, for $\mathrm{BaTiO}_{3}$.

\section{Conclusion}

The problem is solved for a cracked piezoelectric plate. The crack is assumed to be internal, straight and hairline crack. Analytic expressions are obtained for plastic zone length, saturation zone length, crack opening displacement (COD), crack opening potential drop (COP), energy release rate $(J)$. In the case where electrical displacements are set to zero, the solution reduces to the Dugdale model solution for the similar case. Case studies for the qualitative behaviour of COD, COP, $J$ for PZT-4 and $\mathrm{BaTiO}_{3}$ are presented in graphical form for both cases I and II.

Authors are grateful to Prof R D Bhargava (Indian Institute of Technology, Mumbai, India) for his suggestions and encouragement during the course of this work.

\section{References}

Bhargava R R, Saxena N 2005 Solution for a cracked piezoelectric plate subjected to variable load on plastic zones under Mode-I deformation, J. Mater. Process. Technol. 164-165: 1495-1499

Gao C F, Fan W X 1999 A general solution of plane problem in piezoelectric media with collinear cracks. Int. J. Eng. Sci. 37: 347-363

McMeeking R M 2004 The energy release rate for a Griffith crack in a piezoelectric material. Eng. Fracture Mech. 71: 1149-1163

Narita F, Shindo Y 2001 Mode-I crack growth rate for yield strip model of a narrow piezoelectric ceramic body. J. Theor. Appl. Fracture Mech. 36: 73-85

Wang X, Yu S 2001 Transient response of a crack in piezoelectric strip subjected to the mechanical and electrical impacts: Mode-I problem. J. Mech. Mater. 33: 11-20 